\title{
Numerical Analysis of Non-Uniform Heat Source/Sink in a Radiative Micropolar Variable Electric Conductivity Fluid with Dissipation Joule Heating
}

\author{
Rasaq Adekunle Kareem ${ }^{1}$, Sulyman Olakunle Salawu², Jacob Abiodun Gbadeyan ${ }^{3}$ \\ ${ }^{1}$ Department of Mathematics, Lagos State Polytechnic, Ikorodu, Nigeria \\ ${ }^{2}$ Department of Mathematics, Landmark University, Omu-aran, Nigeria \\ ${ }^{3}$ Department of Mathematics, University of Ilorin, Ilorin, Nigeria \\ Email address: \\ raskareem4@yahoo.com(R.A. Kareem), kunlesalawu2@gmail.com(S. O. Salawu),j.agbadeyan@yahoo.com(J. A. Gbadeyan)
}

To cite this article:

Rasaq Adekunle Kareem, Sulyman Olakunle Salawu, Jacob Abiodun Gbadeyan. Numerical Analysis of Non-Uniform Heat Source/Sink in a Radiative Micropolar Variable Electric Conductivity Fluid with Dissipation Joule Heating. American Journal of Applied Mathematics.

Vol. 6, No. 2, 2018, pp. 34-41. doi: 10.11648/j.ajam.20180602.12

Received: February 12, 2018; Accepted: March 6, 2018; Published: March 26, 2018

\begin{abstract}
Computational analysis of radiative heat transfer of micropolar variable electric conductivity fluid with a noneven heat source/sink and dissipative joule heating have been carried out in this article. The flow past an inclined plate with an unvarying heat flux is considered. The transformed equations of the flow model are solved by the Runge-Kutta scheme coupled with shooting method to depict the dimensionless temperature, microrotation and velocity at the boundary layer. The results show that the coefficient of the skin friction and the temperature gradient at the wall increases for regular electric conductivity and non-uniform heat sink/source.
\end{abstract}

Keywords: Radiation, Dissipation, Hydromagnetic, Joule Heating, Micropolar Fluid

\section{Introduction}

A micropolar fluid comprises of gyratory micro-segments that cause fluid to show non-Newtonian activities. The inventions of micropolar fluid flow was discovered useful in exploratory liquid crystals, colloidal suspensions, body fluids, lubricants, turbulent flow of shear, fluid polymeric, preservative suspensions, flows in vessels and microchannels. The theory of micropolar fluids pioneered by [5] has been an energetic research area for long time till present days.

The phenomenons of the model and the utilizations of micropolar fluid was examined by [2], while [9] did tentatively investigation to demonstrate that the present of minute stabilizers polymeric in fluids can diminish the flow impact at the wall up to 25 to 30 percent. The decrease that was depicted by the theory of micropolar substances as announced in [14], the cerebrum fluid that is a case of body fluids can be adequately detailed as micropolar fluids.

Convective flow fluids containing microstructure have many applications, for example, weaken polymer fluids substances, various types of suspensions and fluid gems.
Convective free flow of micropolar fluids past a bended or level surfaces has entranced the psyche of researchers from the time when the flow model was conceived. Many studies have accounted for and examined results on micropolar fluids. Investigated to the impact of radiation on MHD convective heat and mass transfer flow was analyzed by [3, 7]. Also, [1] considered micropolar boundary layer fluid flow along semiinfinite surface utilizing similarity solution to change the models to ordinary different equations. Moreover, [10] reported on the viscous dissipation impacts on MHD micropolar flow with ohmic heating, heat generation and chemical reaction.

Numerous convective flow are brought on by heat absorption or generation which might be as a result of the fluid chemical reaction. The occurrence of heat source or sink can influence the fluid heat profile that modifies the rate of deposition of particle in the structures for example, semiconductor wafers, electronic chips, atomic reactors and so on. Heat absorption or generation has been thought to be temperature dependent heat generation and surface dependent heat generation. The analyzed the effect of non-homogenous 
heat absorption or generation and variable electric conductivity on micropolar fluid was carried out by [15]. It was seen that the plate dependent heat generation is lower contrasted to temperature dependent heat generation. Also, $[11,12]$ carried out study on the influence of thermaldiffusion and non-even heat source/sink on radiative micropolar MHD fluid past a porous medium.

The effect of dissipation on hydromagnetic fluid and heat transfer processes has turned out to be noteworthy in the industry. A lot of engineering practices happen at high temperature with viscous dissipation heat transfer. Such flows have been explored by [17] who examined the influence of viscous dissipation on heat and mass transfer of magnetodyrodynamic micropolar fluid with chemical reaction while [16] considered hydromagnetic micropolar fluid of heat and mass transport in a porous medium with expanding plate, chemical reaction, heat flux and variable micro inertia. In [18], investigation into the MHD Micropolar flow fluid with joule heating, viscous dissipation, constant mass and heat fluxes was carried out. It was noticed from the study that the flow profile rises first within $0 \leq \eta \leq 1$ as the microrotation parameter rises. Afterward, the flow gradually decreases for $\eta>1$ as the microrotation parameter rises. Also, microrotation moves from negative to positive in the boundary layer.

Considering the referred literature, the aim of the present work is to study the viscous dissipation of variable electrically conducting micropolar fluid behavior past an inclined plate in permeable media with thermal radiation, heat fluxes and joule heating for high speed fluid in nonhomogenous heat generation/absorption which have not been considered by many researchers. The study is necessary because of the industrial application of micropolar fluid. Therefore, it is important to study the flow velocity, temperature and microrotation boundary layer at the surface.

\section{The Flow Mathematical Formulation}

Convective flow of two-dimensional viscous, micropolar, laminar fluid through a semi-finite plate that is inclined at an angle $\alpha$ to the vertical is considered. The magnetic field varies in strength as a function of $x$ that is assumed to be in y-direction and defined as $\vec{B}=(0, B(x))$. The Reynolds number is minuet while the outer electric field is assumed as zero. Accordingly, the applied external magnetic field is high contrasted to the stimulated magnetic field. The density $(\rho)$ of the fluid is inert $\left(U_{\infty}=0\right)$ with the buoyancy forces causing the convective motion. The fluid viscosity $\mu$ is considered to be unvarying while the body forces and the pressure gradient are ignored. Rosseland diffusion approximation for radiation is adopted for the flow

Considering the assumptions above, the convective micropolar fluid taking after the Boussinesq approximation may be described by the geometry and subsequent equations.

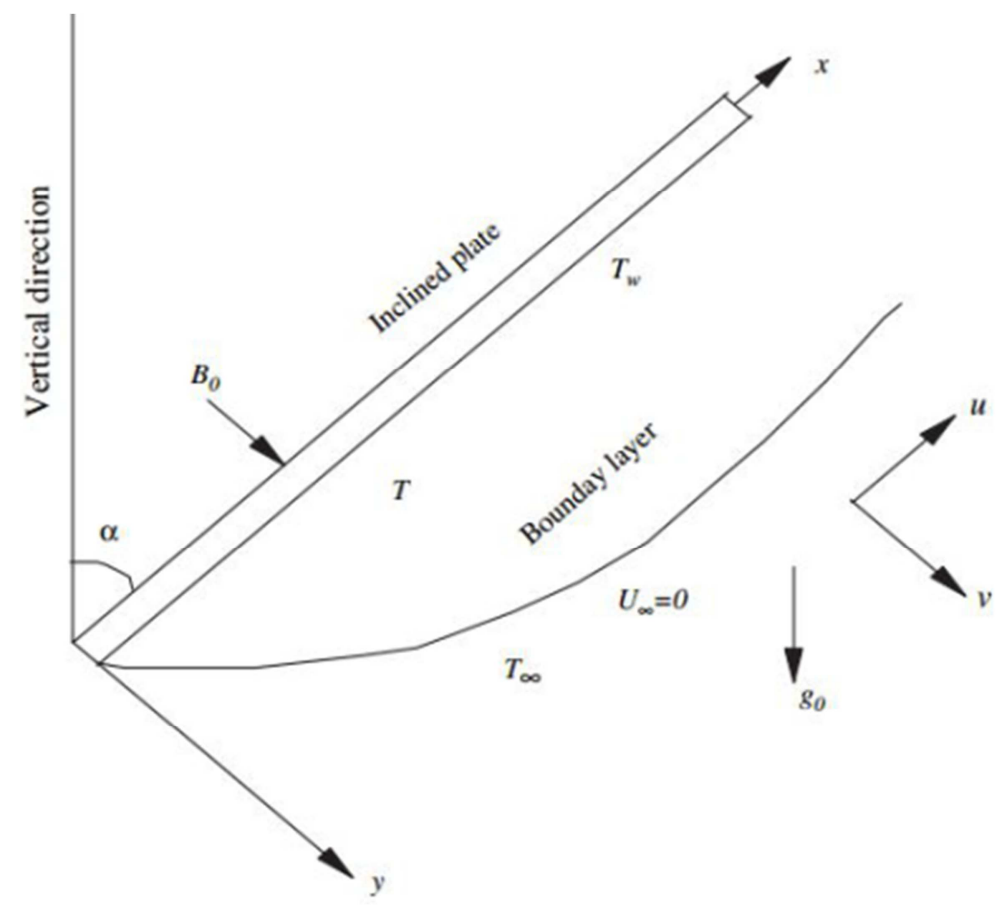

Figure 1. The flow geometry.

$$
\begin{gathered}
\frac{\partial u}{\partial x}+\frac{\partial v}{\partial y}=0 \\
u \frac{\partial u}{\partial x}+v \frac{\partial v}{\partial y}=\left(\frac{\mu+r}{\rho}\right) \frac{\partial^{2} u}{\partial y^{2}}+\frac{r}{\rho} \frac{\partial w}{\partial y}+g_{0} \beta\left(T-T_{\infty}\right) \cos \alpha-\frac{\sigma(B(x))^{2}}{\rho} u-\frac{v}{K} u
\end{gathered}
$$




$$
\begin{gathered}
u \frac{\partial w}{\partial x}+v \frac{\partial w}{\partial y}=\frac{b}{\rho j} \frac{\partial^{2} w}{\partial y^{2}}-\frac{r}{\rho j}\left(2 w+\frac{\partial u}{\partial y}\right) \\
u \frac{\partial T}{\partial x}+v \frac{\partial T}{\partial y}=\frac{k}{\rho c_{p}} \frac{\partial^{2} T}{\partial y^{2}}-\frac{\partial q^{r}}{\partial y}+\left(\frac{\mu+r}{\rho c_{p}}\right)\left(\frac{\partial u}{\partial y}\right)^{2}+\frac{\sigma(B(x))^{2}}{\rho c_{p}} u^{2}+\frac{q^{\prime \prime \prime}}{\rho c_{p}}
\end{gathered}
$$

with the boundary conditions

$$
\begin{aligned}
& u=0, v=0, w=-a \frac{\partial u}{\partial y}, \frac{\partial T}{\partial y}=-\frac{q_{m}}{k} \text { at } y=0 \\
& u=U_{\infty}=0, w=0, T=T_{\infty} \text { as } y \rightarrow \infty
\end{aligned}
$$

where $u$ and $v$ are the fluid velocity in $x$ and $y$ coordinates respectively, $K$ is the permeability of the porous medium, $v=\frac{\mu}{\rho}$ is the kinematic viscosity, $\mu$ is the dynamic viscosity, $\rho$ is the fluid density, $w$ is the microrotaion in $x$ and $y$ components, $b=\left(\mu+\frac{r}{2}\right) j$ is the micropolar viscosity, $j$ is the micro-inertial per unit mass which is assumed to be constant, $r$ is the microrotation coefficient, $k$ is the thermal conductivity, $T$ is the fluid temperature, $c_{p}$ is the specific heat at constant pressure, $g$ is the acceleration due to gravity and $\beta$ is the thermal expansion coefficient.

A linear correlation involving the surface shear $\frac{\partial u}{\partial y}$ and microrotation function $w$ is picked for studying the influence of varying surface circumstances for microroation. Note that the microroation term $a=0$ implies $w=0$, that is the microelement at the wall are not swiveling but while $a=0.5$ implies varnishing of the anti-symmetric module of the stress tensor that stand for feeble concentration. This confirms that for a fine particle suspension at the wall, the particle swivel is the same as the fluid velocity but $a=1$ represents the turbulent boundary layer flows.

Using Rosseland diffusion approximation for radiation $[8,13]$.

$$
q^{r}=-\frac{4 \sigma}{3 \delta} \frac{\partial T^{4}}{\partial y}
$$

where $\sigma$ and $\delta$ are the Stefan-Boltzmann and the mean absorption coefficient respectively, taken the temperature difference in the flow to be sufficiently small such that $T^{4}$ may be consider as a linear function of temperature, introducing Taylor series to expand $T^{4}$ around the free stream $T_{\infty}$ and ignore higher order terms, this gives the approximation

$$
T^{4} \cong 4 T_{\infty}^{3} T-3 T_{\infty}^{4}
$$

Using equation (7), equation (6) can be express as.

$$
\frac{\partial q^{r}}{\partial y}=-\frac{16 \sigma T_{\infty}^{3}}{3 \delta} \frac{\partial^{2} T}{\partial y^{2}}
$$

The non-uniform heat absorption or generation is represented as [11]

$$
q^{\prime \prime}=\frac{k U_{0}}{2 v x}\left[\lambda\left(T-T_{\infty}\right)+\lambda^{*}\left(T_{w}-T_{\infty}\right) e^{-\eta}\right]
$$

where $\lambda^{*}$ and $\lambda$ denote the heat sink/source and space coefficients temperature dependent respectively while $T_{\infty}$ is the free stream temperature. Here, $\lambda>0$ and $\lambda^{*}>0$ stand for heat source but $\lambda<0$ and $\lambda^{*}<0$ depict heat sink.

In this study, it is assumed that the introduced magnetic field strength $B(x)$ is capricious and it is represented as $B(x)=\frac{B_{0}}{\sqrt{x}}$, where $B_{0}$ is constant. Also, the electrical conductivity $\sigma$ depends on the fluid velocity and is defined as $\sigma=\sigma_{0} u$, where $\sigma_{0}$ is constant see Cortell (2007)

Using the following dimensionless variables;

$$
\psi=\sqrt{2 v U_{0} x} f(\eta), \eta=y \sqrt{\frac{U_{0}}{2 v x}}, \theta(\eta)=\frac{T-T_{\infty}}{T_{w}-T_{\infty}}, w=\sqrt{\frac{U_{0}^{3}}{2 v x}} h(\eta)
$$

where $\psi$ is the stream function, $U_{0}$ is the reference velocity

$$
\begin{aligned}
& \text { and } T_{w}-T_{\infty}=\frac{q_{w}}{k \sqrt{\frac{2 v x}{U_{0}}}} \text { while } u=\frac{\partial \psi}{\partial y} \text { and } v=-\frac{\partial \psi}{\partial x} \text { then, } \\
& u=U_{0} f^{\prime}(\eta), \quad v=-\sqrt{\frac{v U_{0}}{2 x}}\left(f(\eta)-\eta f^{\prime}(\eta)\right)
\end{aligned}
$$

Using equations (8) and (9) along with the electric conductivity dependent fluid velocity, variable magnetic field and equation (10) in equations (1)-(5) to obtain,

$$
\begin{gathered}
(1+\delta) f^{\prime \prime \prime}+f f^{\prime \prime}+\delta h^{\prime}+G_{r} \theta \cos \alpha-M f^{\prime 2}-\phi f^{\prime}=0 \\
\left.\varepsilon h^{\prime \prime}-2 \delta\left(2 h+f^{\prime \prime}\right)\right)+\varepsilon\left(f h+g^{\prime} h\right)=0 \\
\left(1+\frac{4}{3} R\right) \theta^{\prime \prime}+(1+\delta) P_{r} E_{c} f^{\prime \prime 2}+M P_{r} E_{c} f^{\prime 3}+P_{r}\left(f \theta^{\prime}-\theta f^{\prime}\right)+\left(\lambda \theta+\lambda^{*} e^{-\eta}\right)=0
\end{gathered}
$$


The boundary conditions become

$$
\begin{aligned}
& f=f^{\prime}=0, h=-a f^{\prime \prime}, \theta^{\prime}=-1 \text { at } \eta=0 \\
& f^{\prime}=0, h=0, \theta=0 \text { as } \eta \rightarrow \infty
\end{aligned}
$$

where $\delta=\frac{r}{\mu}$ is the vortex viscosity term, $M=\frac{2 \sigma_{0} B_{0}^{2}}{\rho}$ is the magnetic field term, $\phi=\frac{2 x v}{U_{0} K}$ is the permeability parameter, $\varepsilon=\frac{j U_{0}}{v x}$ is the micro-inertia density term, $R=\frac{4 \sigma T}{\delta k}$ is the radiation parameter, $E_{c}=\frac{U_{0}^{2}}{\left(T_{w}-T_{\infty}\right) c_{p}}$ is the Eckert number, $G_{r}=\frac{2 g x \beta\left(T_{w}-T_{\infty}\right)}{U_{0}^{2}}$ is the thermal Grashof number and $P_{r}=\frac{\mu c_{p}}{k}$ is the Prandtl number.

The essential engineering quantities of interest for this flow are the local skin friction $C_{f}$ and Nusselt number $N_{u}$ given as:

$$
C_{f}=\frac{2 \tau_{w}}{\rho u_{w}^{2}}, \quad N u=\frac{x q_{w}}{k\left(T_{w}-T_{\infty}\right)}
$$

$\tau_{w}$ and $q_{w}$ are respectively taken as

$$
\tau_{w}=\mu\left(\frac{\partial u}{\partial y}\right)_{y=0}, q_{w}=k\left(\frac{\partial T}{\partial y}\right)_{y=0}
$$

Therefore,

$C_{f}=\left(2 \operatorname{Re}_{x}^{-1}\right)^{\frac{1}{2}}[1+(1-a) \delta] f^{\prime \prime}(0), \quad N u_{x}=\left(2^{-1} R e_{x}\right)^{\frac{1}{2}} \frac{1}{\theta(0)}$

The computational values for $C_{f}$ and $N u_{x}$ are obtained from equations (18)

Table 1. Comparison of $f^{\prime \prime}(0)$ for $\delta=\varepsilon=G_{r}=\alpha=R=E_{c}=0$, various values of $a$

\begin{tabular}{lllll}
\hline $\boldsymbol{a}$ & {$[\mathbf{4}]$} & {$[\mathbf{6}]$} & {$[\mathbf{1 5}]$} & Present results \\
\hline 0.0 & 0.627547 & 0.627555 & 0.627498 & 0.623534 \\
0.2 & 0.766758 & 0.766837 & 0.767066 & 0.766831 \\
0.5 & 0.889477 & 0.889544 & 0.892366 & 0.891784 \\
0.75 & 0.953786 & 0.953975 & 0.956365 & 0.954523 \\
1.0 & 1.000000 & 1.000000 & 1.002125 & 1.001986 \\
\hline
\end{tabular}

Table 2. Values of $f^{\prime \prime}(0)$ and $\theta^{\prime}(0)$ for different values of $M, E_{c}, \lambda, \lambda^{*}, \phi$, and $\delta$ on PP-Physical Parameters.

\begin{tabular}{llllllll}
\hline$P P$ & values & $\boldsymbol{f}^{\prime \prime}(0)$ & $\boldsymbol{\theta}^{\prime}(0)$ & $\boldsymbol{P P}$ & values & $\boldsymbol{f}^{\prime \prime}(0)$ & $\boldsymbol{\theta}^{\prime}(0)$ \\
\hline$M$ & 1 & 1.11795 & 6.33597 & $R$ & 0.1 & 1.01758 & 5.36740 \\
& 3 & 1.15638 & 7.72407 & & 0.5 & 1.08877 & 5.55485 \\
& 5 & 1.19926 & 8.96274 & & 0.7 & 1.15301 & 5.84632 \\
$E_{c}$ & 7 & 1.24187 & 10.11891 & & 1.0 & 1.20395 & 6.03903 \\
& 0.2 & 1.11101 & 5.94768 & $\phi$ & 0.007 & 1.11204 & 5.67261 \\
& 0.5 & 1.17319 & 6.35698 & & 0.1 & 1.11127 & 5.80543 \\
& 0.7 & 1.21929 & 6.66562 & & 0.3 & 1.11126 & 6.08932 \\
& 1.0 & 1.29671 & 7.19343 & & 0.5 & 1.11303 & 6.37080 \\
& 0.0 & 0.52826 & 2.79851 & $\lambda^{*}$ & 0.0 & 1.01758 & 5.36740 \\
& 0.1 & 0.60853 & 3.19403 & & 0.5 & 1.11101 & 5.94768 \\
& 0.3 & 0.82009 & 4.29704 & & 1.0 & 1.19538 & 6.48375 \\
\hline
\end{tabular}

\section{Results and Discussion}

The computational outcomes for the coupled differential equations are gotten for the dimensionless microrotation, temperature and velocity profiles. In the analysis, the default parameters value are taken as: $a=0.5, P_{r}=0.73, G_{r}=2.5, \beta=0.2, \varepsilon=2, R=0.1, E_{c}=0.2$, $M=0.5, \alpha=30^{\circ}, \delta=1, \lambda=0.5$ and $\lambda^{*}=0.5$. The values depend on the decision of existing articles in view of inaccessibility of investigation figures for vortex viscosity and micro-inertia density parameters, appropriate values are chosen to confirm the polar impact on flow properties.
Table 1 depicts the computational outcomes that demonstrate the action of microrotation parameter $a$ on the fluid flow parts of the present result contrasted with the existing results. The comparison is observed to be in a superb agreement as appeared in the table.

Table 2 represents the computational results, that show the influence of some fluid properties on the flow and heat part of the investigation. It is noticed from the table that an increase in the values of parameters $M, E_{c}, \lambda, \lambda^{*}$ and $\beta$ enhances the skin friction and the temperature gradient at the plate due to an increase in the flow and temperature boundary layer thickness. Additionally, an increase in values of $\delta$ causes shrinking in the flow at the plate but enhances the 
thermal gradient at the surface.

Figures 2 and 3 portray the fluid flow and microrotation profiles for various values of the magnetic field term $M$. It is seen from Figure 2 that the flow diminishes as the values of $M$ increases because of the influence of Lorentz forces that retard the convective fluid motion. Figure 3, affirmed that as the values of the magnetic field parameter increases the microrotation near the plate within the range $0 \leq \eta \leq 1.2$ increases but reduces as it distance away from the surface within the range $1.2 \leq \eta \leq 5$. The outcomes demonstrate that the magnetic field might be use to maintain the flow and heat transfer properties.

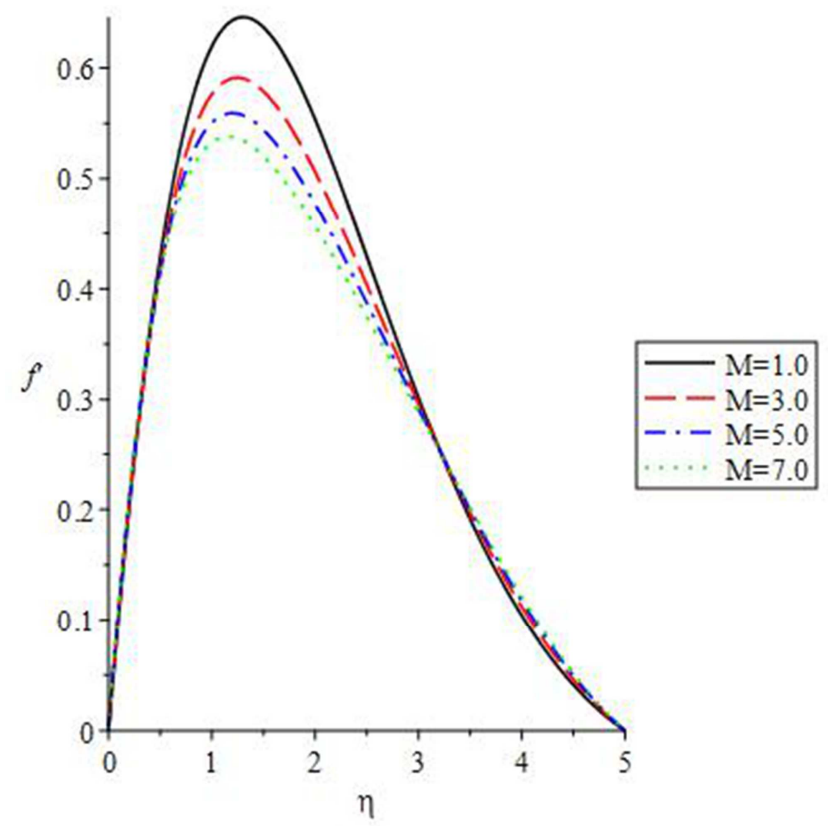

Figure 2. Velocity fields for different values of $M$.

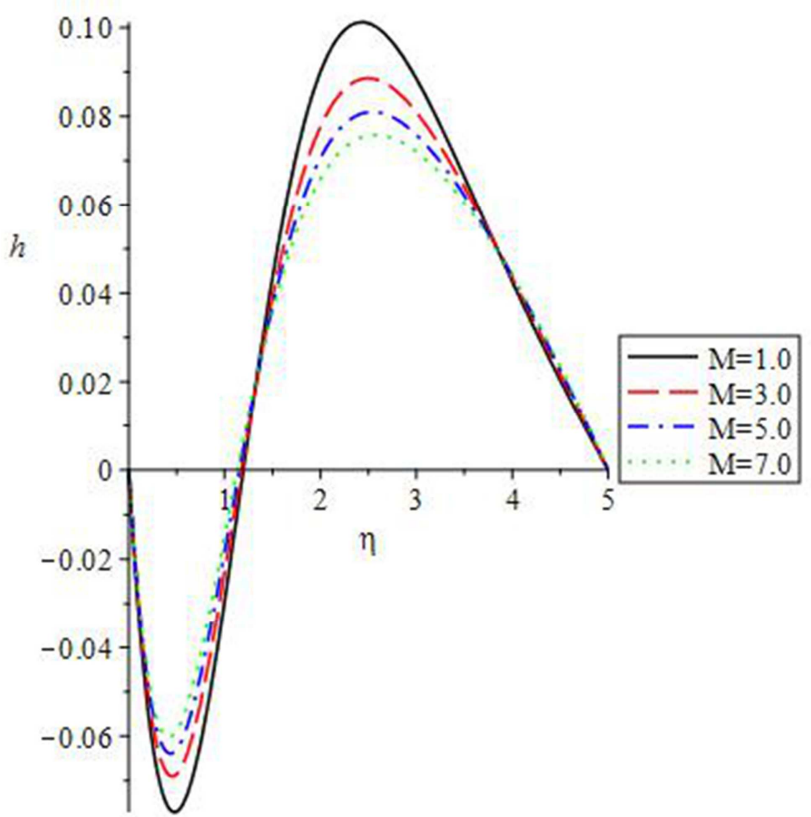

Figure 3. Microrotation fields for different values of $M$.
Figures 4 and 5 show the effect of the vortex viscosity $\delta$ on the fluid velocity and microrotation field. It is found that a rise in the values of $\delta$ initial abatement the fluid velocity at $\eta \leq 1.6$ but increases at range $1.6 \leq \eta \leq 5$ while microrotation profiles near to the surface at $\eta \leq 2$ decreases and later increases as it moves away from the surface. The reason is because the viscous friction within the fluid tends to organize the flow into a collection of irrotational vortices and a moving vortex carries with it some angular and linear momentum and energy.

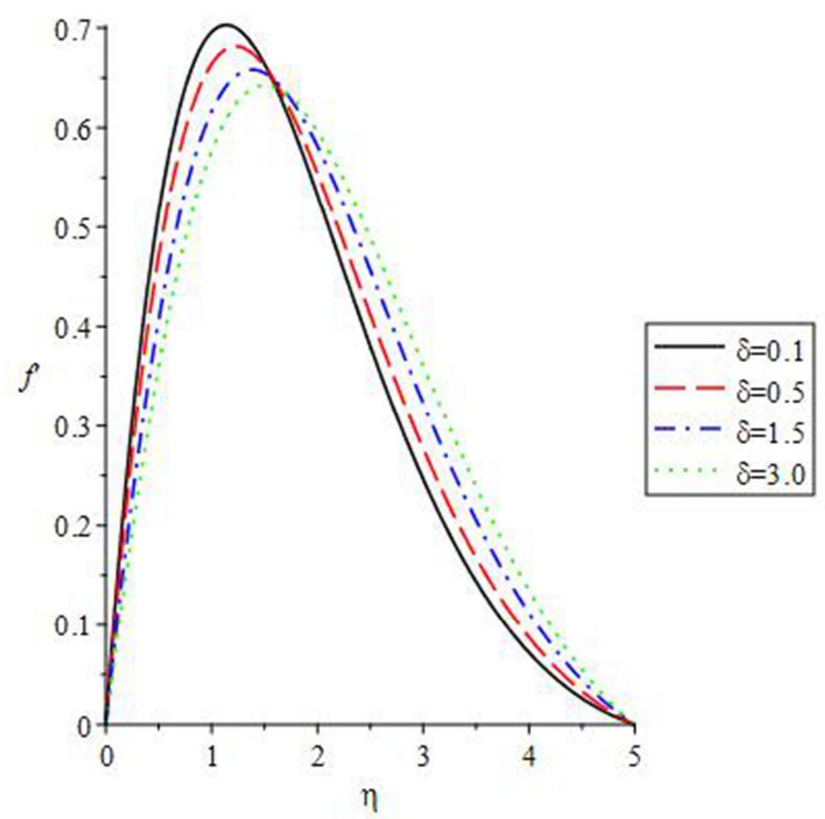

Figure 4. Velocity fields for different values of $\delta$.

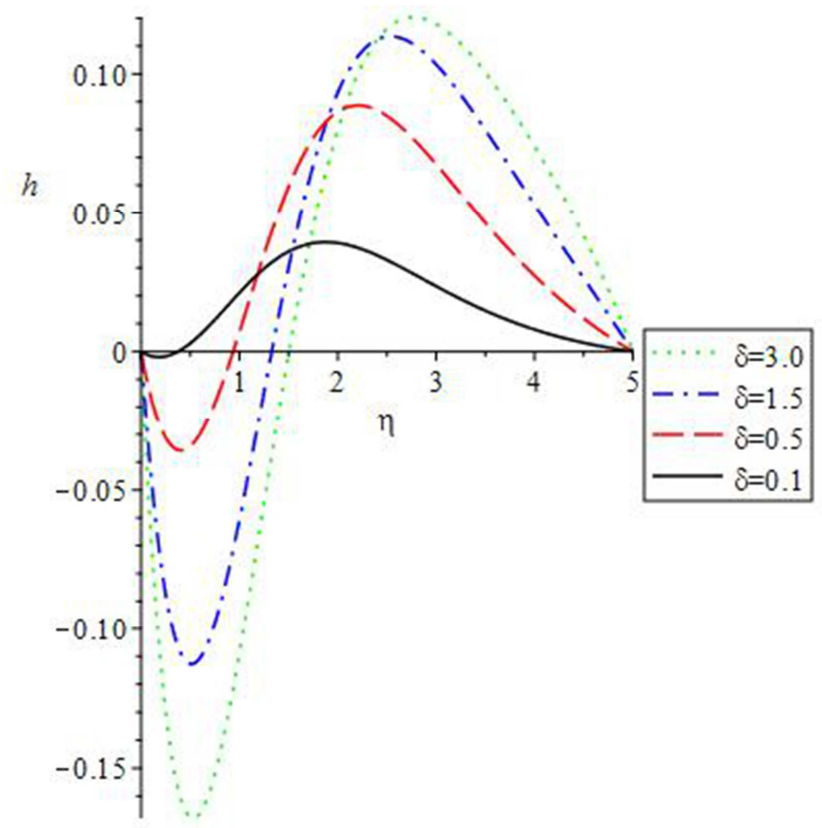

Figure 5. Microrotation fields for different values of $\delta$.

Figures 6-9 represent the microrotation and temperature fields for different values of temperature dependent and 
surface temperature heat absorption or generation terms $\lambda$ and $\lambda^{*}$. Figure 6 and 8 demonstrated that a rise in the values of $\lambda$ and $\lambda^{*}$ decreases the microrotation near the surface at $\eta \leq 1.5$ and it increases as it far away from the surface. Also, in Figures 7 and 9 the temperature distribution increases as the parameters $\lambda$ and $\lambda^{*}$ increases respectively. This is because the transfer of thermal energy across a well-defined boundary decreases that it in turn increases the amount of heat within the system and thereby causes rise the profiles.

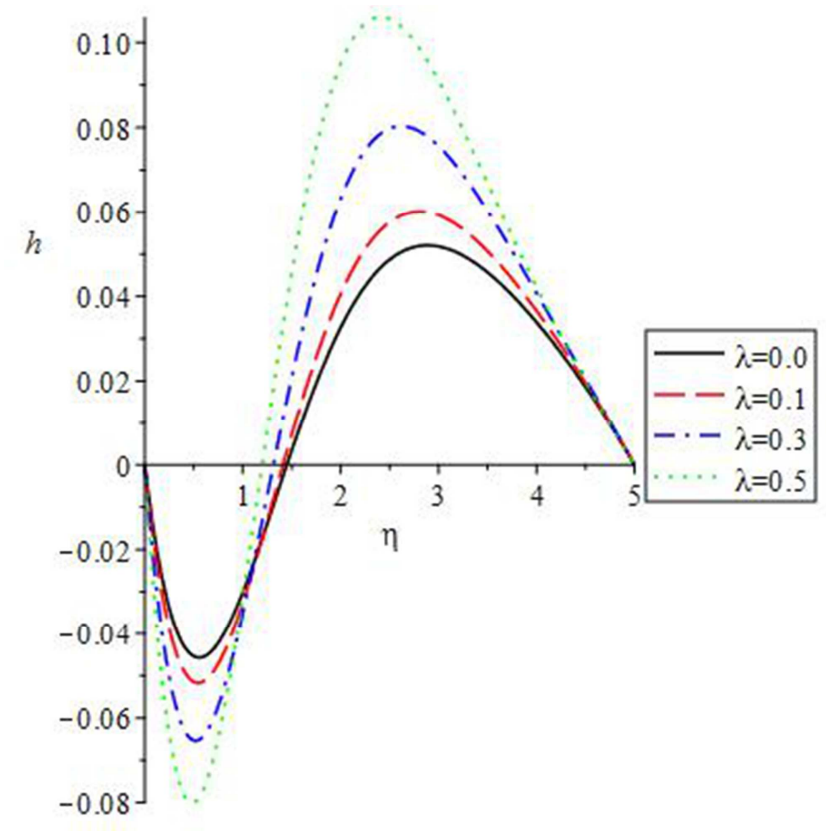

Figure 6. Microrotation fields for different values of $\lambda$.

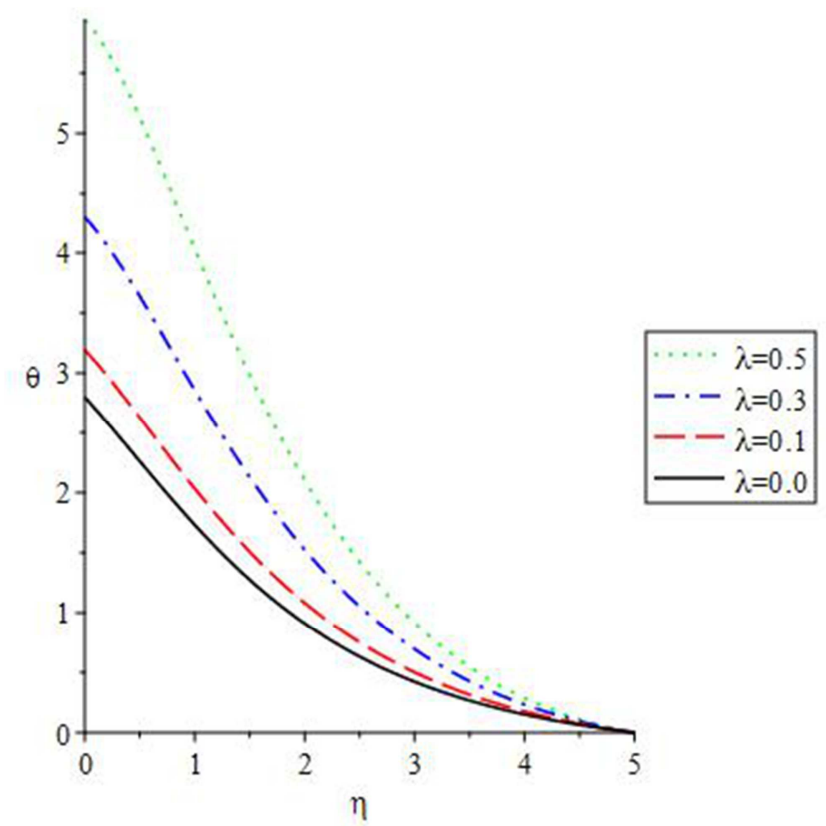

Figure 7. Temperature fields for different values of $\lambda$.

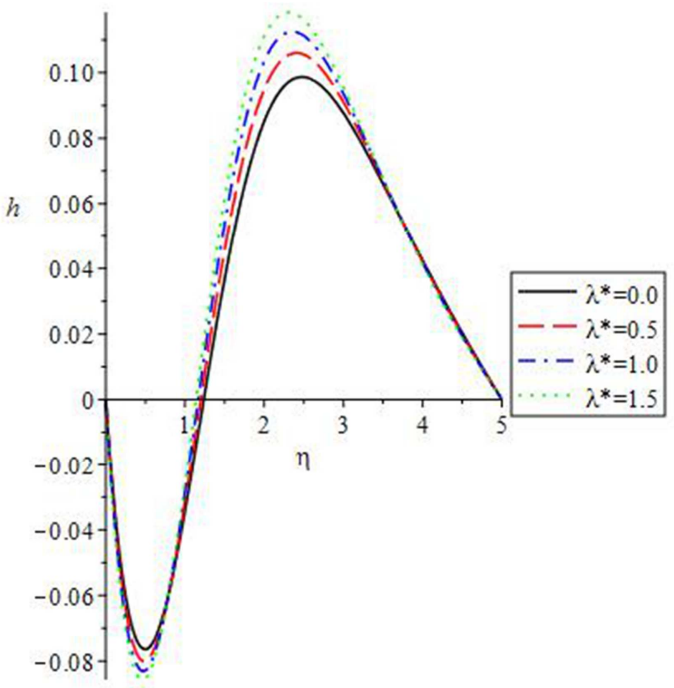

Figure 8. Microrotation fields for different values of $\lambda^{*}$.

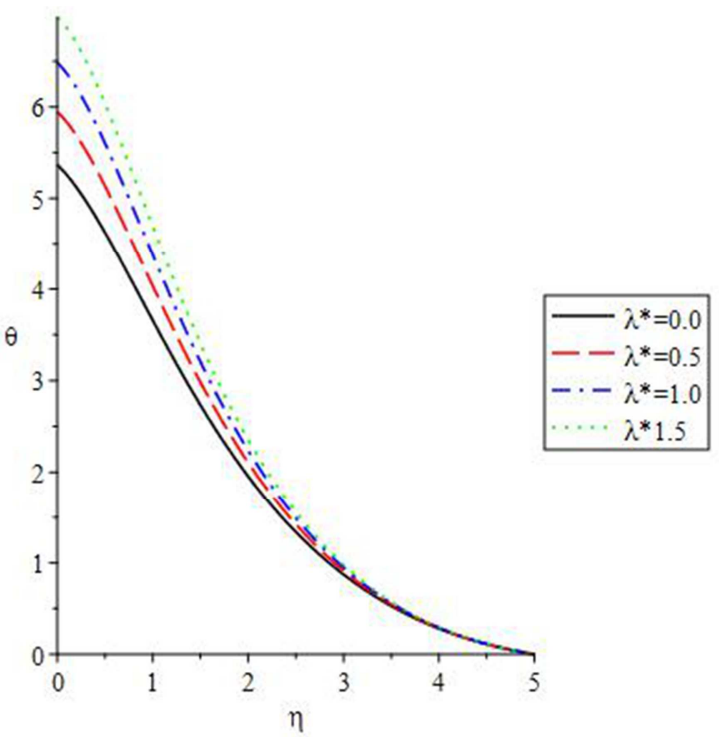

Figure 9. Temperature fields for different values of $\lambda^{*}$.

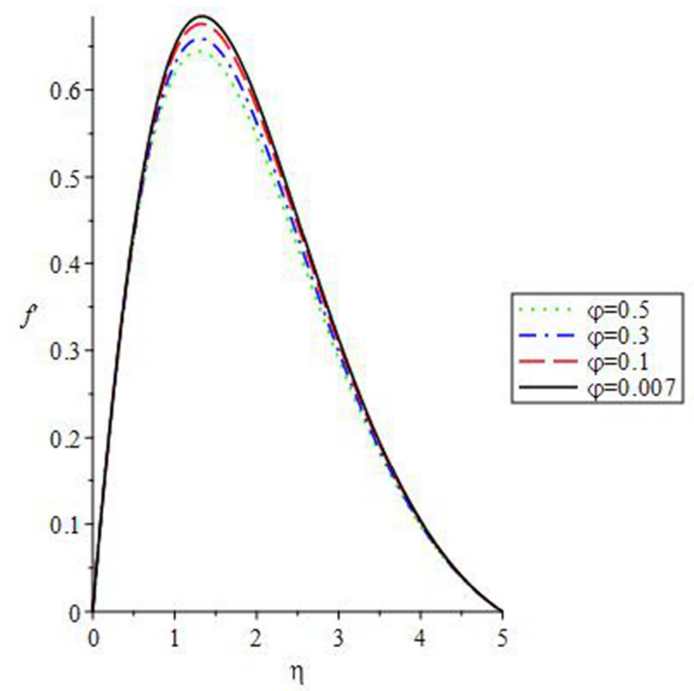

Figure 10. Velocity fields for different values of $\phi$. 
Figure 10 depicts the effect of porosity term $\phi$ on the dimensionless velocity. It is evidenced that the flow field decreases as the porosity parameter rises. This is as a result of the wall of the plate that gives an additional opposition to the flow mechanism by influencing the fluid to move at a decelerated rate.

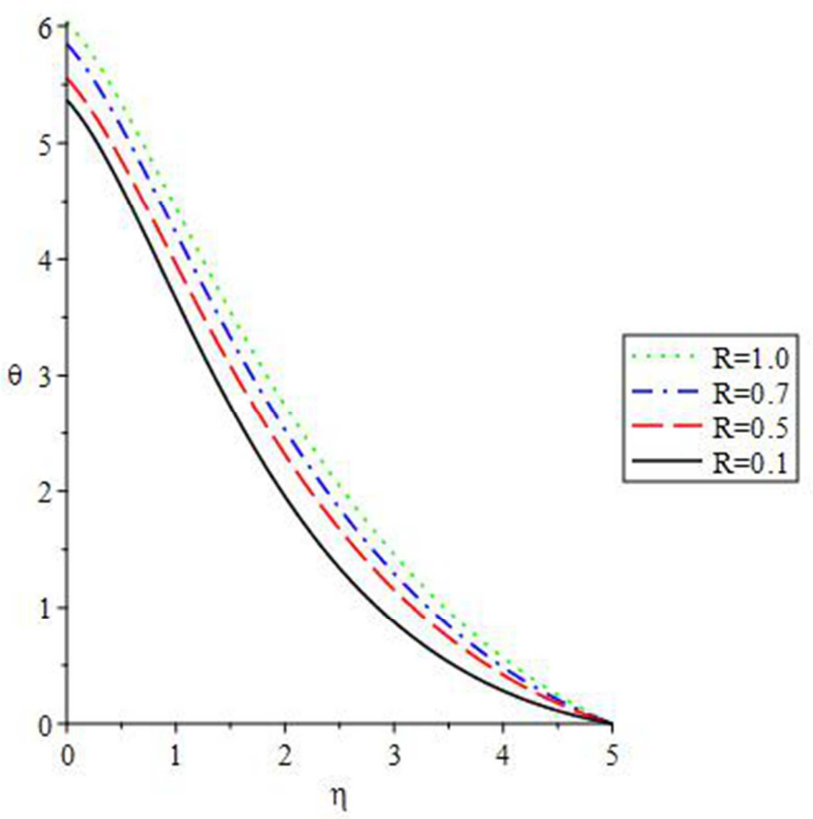

Figure 11. Temperature fields for different values of $R$.

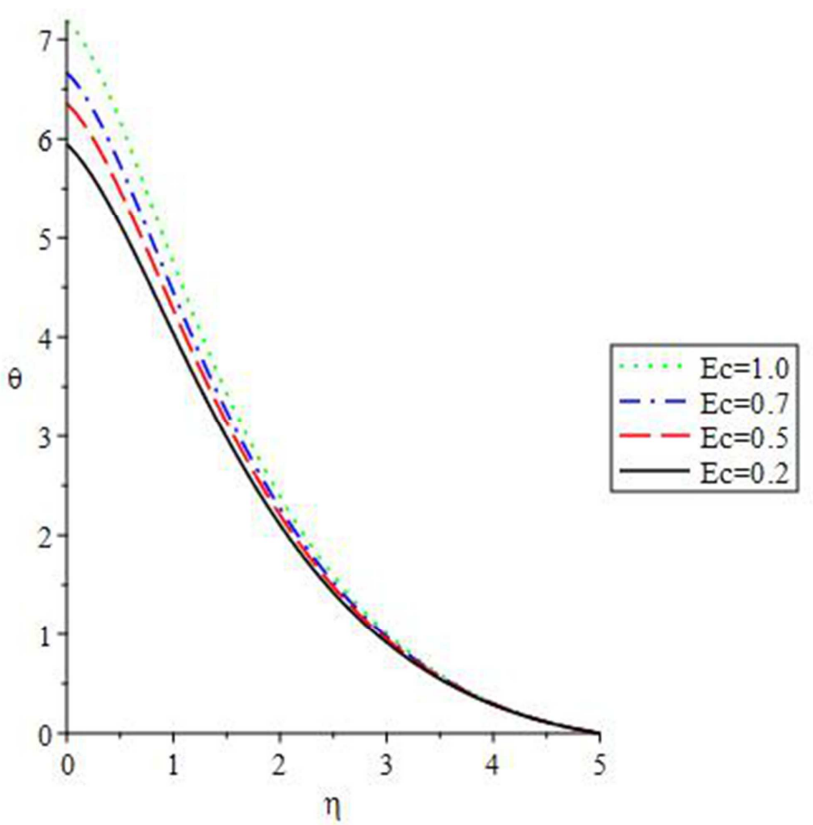

Figure 12. Temperature fields for different values of $E_{c}$.

The influence of radiation on the temperature profiles is given in figure 11. It is noticed that as the values of $R$ increases, there is corresponding enhancement in the temperature fields which results in an increase in the thermal boundary layer thickness and thereby decreases the amount of emission or transmission of energy in the form of waves or particles through space or through a material medium out of the system.

Figure 12 represent the consequent of variation in the Eckert number $E_{c}$ on the temperature distribution. Eckert number expresses the relationship between kinetic energy and enthalpy difference, and is used to characterize heat dissipation. It is noticed that the temperature field increases with a rise in the values of $E_{c}$. This is due to increase in the temperature boundary layer thickness that reduces the amount of energy dissipating out of the system and thereby causes a rise in the heat distribution

\section{Conclusion}

Numerical simulation was carried out for dimensionless boundary layer equations of convective heat transfer of a hydromagnetic and micropolar fluid past inclined surface with non-uniform heat sink/source. It is observed from the study that, the magnetic field decreases the flow rate and angular velocity while the temperature dependent and surface dependent temperature heat sink or source parameters as well as viscous dissipation parameter increases angular velocity and temperature distributions. The vortex viscosity parameter decreases the flow and microrotation profiles near the wall.

\section{References}

[1] Ahmadi, G. (2013). Self-similar solution of incompressible micropolar boundary layer flow over a semi-infinite plate, Int. J. Eng. Sci., 14, 639-646.

[2] Ariman, T., Turk, M. A., Sylvester, N. D. (1974). Microcontinuum fluid mechanics, a review. Int. J. Eng. Sci. 12, 273.

[3] Bhuvaneswari, M. Sivasankaran, S., Kim, Y. J. (2010). Exact analysis of radiation convective flow heat and mass transfer over an inclined plate in porous medium, World Appl. Sci. J., 7, 774-778.

[4] Cortell, R. (2007). Viscous flow and heat transfer over a nonlinearly stretching sheet. Appl. Math. Comput., 184, 864873.

[5] Eringen, A. C. (1966). Theory of micropolar fluids, J. Math. Mech., 16, 1-18.

[6] Hayat, T., Abbas, Z., Javed, T. (2008). Mixed convection flow of micropolar fluid over a nonlinearly stretching sheet. Phys. Lett. A, 372, 637-647.

[7] Hayat, T., Qasim, M. (2010), Influence of thermal radiation and Joule heating MHD flow of a Maxwell fluid in the presence of thermophoresis, Int. J. Heat Mass Transfer, 53, $4780-4788$.

[8] Helmy, K. A. (1995). MHD boundary layer equations for power law fluids with variable electric conductivity. Meccanica, 30, 187-200.

[9] Hoyt, J. W., Fabula, A. G. (1964). The Effect of additives on fluid friction, US Naval Ordnance Test Station Report. 
[10] Khilap, S., Manoj, K. (2015). Effect of viscous dissipation on double stratified MHD free convection in micropolar fluid flow in porous media with chemical reaction, heat generation and ohmic Heating, Chemical and Process Engineering Research, 31, 75-80.

[11] Mabood, F., Ibrahim, S. M. (2016). Effects of soret and nonuniform heat source on MHD non-Darcian convective flow over a stretching sheet in a dissipative micropolar fluid with radiation, Journal of Applied Fluid Mechanics, 9, 2503-2513.

[12] Mabood, F., Ibrahim, S. M., Rashidi, M. M., Shadloo, M. S., Giulio L. (2016). Non-uniform heat source/sink and Soret effects on MHD non-Darcian convective flow past a stretching sheet in a micropolar fluid with radiation, International Journal of Heat and Mass Transfer, 93, 674-682.

[13] Mebarek-Oudina, F., Bessaih, R. (2014). Numerical modeling of MHD stability in a cylindrical configuration. Journal of the Franklin Institute, 351, 667-681.

[14] Power, H. (1998). Micropolar fluid model for the brain fluid dynamics, in: Intl. Conf. on bio-fluid mechanics, U.K.
[15] Rahman, M. M., Uddin, M. J., Aziz, A. (2009). Effects of variable electric conductivity and non-uniform heat source (or sink) on convective micropolar fluid flow along an inclined flat plate with surface heat flux, Int. Journal of Thermal Sciences, 48, 2331-2340.

[16] Rawat, S., Kapoor, S., Bhargava, R. (2016). MHD flow heat and mass transfer of micropolar fluid over a nonlinear stretching sheet with variable micro inertia density, heat flux and chemical reaction in a non-darcy porous medium, Journal of Applied Fluid Mechanics, 9, 321-331.

[17] Siva, R. S., Shamshuddin, M. D. (2015). Heat and mass transfer on the MHD flow of micropolar fluid in the presence of viscous dissipation and chemical reaction, Int. Conference on computational Heat and Mass transfer, 127, 885-892.

[18] Ziaul Haquea, Md., Mahmud Alam, Md., Ferdows, M., Postelnicu, A. (2012). Micropolar fluid behaviors on steady MHD free convection and mass transfer flow with constant heat and mass fluxes, joule heating and viscous dissipation, Journal of King Saud University Engineering Sciences, 24, 71-84. 